performed on the residuals using standard Bioconductor packages (limma, minfi and lumi). Epithelial cell content differences were accounted for in our analysis. To facilitate generalisation of results, this process was repeated in a manner similar to hold-out cross validation, where a sub-cohort generates a discovery model, and the remaining samples are used to test it. This was repeated 1000 times. The loci that were the most often selected were used in a Random Forest machine-learning algorithm to assess their classification performance.

Results The 50 most frequently selected probes showed a noticeable pattern of global separation between controls and Crohn's samples in both discovery and test sets and have clear biological relevance including reported effects on NOD2 signaling and Th1/Th17 balance. Diagnostic accuracy for a 5 probe classifier was $72 \%$, rising to $79 \%$ with 10 probes and $90 \%$ when 40 probes were included. The dataset, though matched for cases and controls, has a wide distribution of ages (10 - 87 years old), and includes both sexes (in roughly a 2-to-1 female-to-male ratio). Location of Crohn's in the gut also varies between participants. This is a modest result that is nonetheless strongly suggestive that saliva contains valuable information about the disease status of the individual even when applied on the most general of cohorts. Further refinement of the test cohort or the use of this result with other clinical data has the potential to increase this accuracy and make this a viable diagnostic tool in the future.

Conclusion We have preliminary evidence that a non-invasive saliva-based candidate biomarker panel could be implemented to offer a cheap, rapid, self-administered screening method for OAC.

\section{PMO-4 IMMUNE CHECKPOINT INHIBITOR-INDUCED COLITIS IS MEDIATED BY IL23 RESPONSIVE CD90+ CYTOTOXIC LYMPHOCYTES}

${ }^{1}$ Jonathan Lo*, ${ }^{2}$ Domenico Cozzetto, ${ }^{2}$ Jillian Yong Xin Sieh, ${ }^{3}$ Matthew Madgwick, ${ }^{1}$ Hiromi Kudo, 'James Alexander, 'Jesus Miguens-Blanco, ${ }^{3}$ Tamas Korcsmaros, ${ }^{1}$ Robert Goldin, ${ }^{1} J u l i a n$ Marchesi, ${ }^{4}$ Graham Lord, ${ }^{1}$ Nick Powell. ${ }^{1}$ Imperial College London, London, UK; ${ }^{2}$ King's College London, London, UK; ${ }^{3}$ Earlham Institute, Norwich, UK; ${ }^{4}$ University of Manchester, Manchester, UK

\subsection{6/gutjnl-2021-BSG.143}

Background and aims Immune checkpoint inhibitors (CPI) have revolutionised cancer treatment, with previously untreatable disease now amenable to potential cure. Combination regimens of anti-CTLA4 and anti-PD-1 show enhanced efficacy but are prone to off target immune-mediated tissue injury, particularly at the barrier surfaces. CPI-induced colitis is a common a serious complication.

Methods To probe the impact of immune checkpoints on intestinal homeostasis mice were challenged with combination anti-CTLA4 and anti-PD-1 immunotherapy, manipulation of the intestinal microbiota and antibody blockade/depletion studies. Colonic immune responses were profiled using RNAsequencing, including high-resolution single cell analyses, and flow cytometry.

Results CPI colitis was dependent on the composition of the intestinal microbiota and was characterized by remodelling of mucosal lymphocytes with induction of polyfunctional, cytolytic responses in T-cells (both $\mathrm{CD}^{+}$and $\mathrm{CD}^{+}$cells) and innate lymphoid cells (ILCs), all of which likely participated in immune-mediated tissue injury. $\mathrm{CD}^{+} 0^{+}$lymphocytes were especially enriched for cytolytic molecules (Gzmb, Prf1, Nkg7), pro-inflammatory cytokines (Ifng, Il22 and Il17a), and chemokines (Ccl3, Ccl4 and Ccl9). Network analysis of predicted upstream regulators identified multiple potential activators of $\mathrm{CD}^{+}$mucosal lymphocytes, including IL23. Functionally, CD90 depletion or IL23 blockade significantly attenuated CPIcolitis.

Conclusions This study provides new mechanistic insights into CPI colitis, identifying IL23 responsive $\mathrm{CD}^{+} 0^{+}$lymphocytes with cytolytic and polyfunctional cytokine responses as key mediators of disease. Therapeutic targeting of these pathogenic effector cells likely holds the key to preventing and reversing CPI colitis.

\section{PMO-5 STEROIDS AND TAPERING REGIMES IN INFLAMMATORY BOWEL DISEASE - WHAT DO WE PRESCRIBE?}

Joseph Salem*, Richard Pollok. St George's Hospitals NHS Foundation Trust, London, UK

\subsection{6/gutjnl-2021-BSG.144}

Introduction Steroids have been an established treatment in flares of inflammatory bowel disease (IBD) for the past 50 years. Whilst evidence exists to guide the initial dose of these drugs, data regarding the optimal length and tapering regime is lacking and consequently anecdotal reports suggests prescribing practice varies substantially. Steroids have a wide range of side-effects according to the dose and duration of therapy. We aimed to help characterise steroid prescribing practice in the UK for the management of inflammatory bowel disease.

Methods A survey was created using Google Forms ${ }^{\oplus}$ and circulated with the support of the British Society of Gastroenterology using the members email distribution over a 2 week period. All questions were mandatory and focused around clinical scenarios of mild-to-moderate disease flares in Ulcerative Colitis (UC) and Crohn's Disease (CD).

Results 128 healthcare professionals completed the survey of which 123 were able to prescribe steroids. 80\% of respondents were consultants $(n=98)$ followed by $10 \%$ specialist registrar $(n=12)$ and 4\% IBD CNS $(n=5)$.

$92 \%$ of prescribers $(n=113)$ would treat a UC flare with $40 \mathrm{mg}$ Prednisolone. 65\% $(\mathrm{n}=80)$ would give full treatment dose for 7 days followed by $23 \%(n=28)$ giving full treatment for 14 days. $86 \%(n=106)$ would prescribe a tapering regime of $5 \mathrm{mg}$ every week with $7 \%(\mathrm{n}=8)$ tapering $5 \mathrm{mg}$ every 5 days. $98 \%$ of respondents $(n=121)$ would not prescribe steroids to maintain remission of UC.

90\% of prescribers $(n=111)$ would give the same dose and taper for a Crohn's patient with a similar flare. For those prescribing differently in a CD flare, $67 \%(n=8)$ would start at Prednisolone $40 \mathrm{mg}$ followed by $17 \%(\mathrm{n}=2)$ prescribing a dose of $0.75-1 \mathrm{mg} / \mathrm{kg}$ and $17 \%(\mathrm{n}=2)$ a differing preparation. $50 \%$ $(n=6)$ would prescribe full treatment dose for 7 days followed by $25 \%(n=3)$ prescribing full treatment for 14 days and $17 \% \quad(n=2)$ prescribing full treatment for greater than 14 days. $50 \%(n=6)$ would prescribe a tapering regime of $5 \mathrm{mg}$ every week with $33 \%(\mathrm{n}=4)$ tapering $5 \mathrm{mg}$ every 5 days.

$72 \%$ prescribed 2 nd generation synthetic steroids (budesonide preparations/beclomethasone) for UC patients $(n=88)$ versus $76 \%$ in $C D$ patients. $54 \% \quad(n=66)$ would use these preparations before conventional steroids in mild-moderate flares of UC versus $79 \%$ in mild-moderate flares of CD. 
Conclusion The survey indicates some variation in steroid prescribing for IBD patients. The majority prescribe an 8 week regime with a starting dose of $40 \mathrm{mg}$ daily for 7 days and a taper of $5 \mathrm{mg}$ every week thereafter. 2nd generation steroids were more frequently prescribed in CD than in UC with the majority agreeing that these preparations should be offered before conventional steroids. Whilst steroid prescribing is not underscored by a firm evidence base it is reasonably consistent. Further research is needed to define the optimal tapering regime.

\section{PMO-6 IMPACT OF CONSULTATION FREQUENCY AND TIME TO DIAGNOSIS ON SUBSEQUENT INFLAMMATORY BOWEL DISEASE OUTCOMES}

\footnotetext{
${ }^{1,2}$ Nishani Jayasooriya* ${ }^{2} S$ Saxena, ${ }^{1,2}$ J Blackwell, ${ }^{3}$ I Petersen, ${ }^{2} \mathrm{~A}$ Bottle, ${ }^{2} \mathrm{H}$ Creese, 1,2R Pollok. 'Gastroenterology, St George's University of London, UK; ${ }^{2}$ Dept. Primary Care and Public Health, Imperial College London, UK; ${ }^{3}$ Institute of Epidemiology and Health, University College London, UK
}

\subsection{6/gutjnl-2021-BSG. 145}

Introduction The impact of length of time between the point of initial contact, at symptom onset, with a health-care provider and diagnosis of inflammatory bowel disease (IBD) on subsequent disease outcomes is unclear. Diagnosis can be challenging and delay common, with an excess of gastrointestinal (GI) symptoms reported 3 years before diagnosis of IBD compared to the background population. ${ }^{1}$

We describe the impact of time to diagnosis and frequency of consultation amongst individuals with GI symptoms who later go on to develop ulcerative colitis (UC) and Crohn's disease (CD).

Methods Using the Clinical Practice Research Datalink, a nationally representative research database, incident cases of IBD were identified between 2003 and 2016. GI symptoms were defined as abdominal pain, diarrhoea or rectal bleeding. Proportion of individuals consulting for incident GI symptoms within 3 years prior to IBD diagnosis was identified. Using a multivariable regression model we evaluated the association between time to diagnosis from incident consultation and consultation frequency for GI symptoms on disease outcomes (corticosteroid (CS) and thiopurine (TP) use, hospitalisation and major abdominal surgery) 5 years after IBD diagnosis.

Results 6,967 incident cases of IBD were identified during the study period. Within 3 years prior to IBD diagnosis, 2,645 (38\%) patients had an incident presentation with GI symptoms in primary care $(782 \mathrm{CD}, 1,863 \mathrm{UC})$. Presentation with GI symptoms occurred $>3$ years before IBD diagnosis in 2,842 $(41 \%)$ of patients. There was no recorded primary care consultation for $1,480(21 \%)$ patients.

Time to diagnosis from initial consultation was not associated with worse subsequent disease outcomes. However, amongst patients later diagnosed with $U C, \geq 3$ prior consultations for GI symptoms was associated with an increased subsequent risk of CS use (HR 1.19, 95\% CI 1.05 -1.36), CS dependency (HR 1.50, 95\% CI 1.10 -2.05), TP use (HR $1.60,95 \%$ CI $1.22-2.11$ ) and colectomy (HR 1.91, 95\% CI 1.21 - 3.04). Amongst patients with $C D, \geq 3$ prior consultations was associated with an increased subsequent risk of major abdominal surgery (HR 1.75 , 95\% CI $1.22 \quad-2.5$ ) and hospitalisation (HR 1.58, 95\% CI 1.18 -2.11)

Conclusion Frequent primary care consultation with GI symptoms, but not symptom duration prior to IBD diagnosis, was associated with worse subsequent disease outcomes. Steps are needed to expedite IBD diagnosis to reduce the risk of adverse disease outcomes.

\section{REFERENCE}

1. Blackwell, J. et al. Prevalence and Duration of Gastrointestinal Symptoms Before Diagnosis of Inflammatory Bowel Disease and Predictors of Timely Specialist Review: A Population-Based Study. J. Crohn's Colitis (2020)

\section{PM0-7 PATIENT-REPORTED OUTCOMES ON THE IMPACT OF ORAL STEROID TREATMENT IN ULCERATIVE COLITIS}

Nishani Jayasooriya, Jonathan Blackwell, Richard Pollok. Dept. Gastroenterology St George's Hospitals NHS Foundation Trust, London, UK

\subsection{6/gutjnl-2021-BSG.146}

Introduction Corticosteroids (CS) have formed the main stay of therapy for the treatment of acute flares of ulcerative colitis (UC). ${ }^{1}$ Data regarding the optimal tapering regime however is lacking. Since the introduction of CS it became apparent that their use is often associated with short and long-term side effects. ${ }^{2}$ Likewise rapid discontinuation of CS may also cause withdrawal symptoms. There remains a gap in the evidence base defining the optimal oral CS tapering regime to ensure adequate efficacy while minimising undue side effects

We aimed to explore patient perspective on the general impact, benefits and side effects, associated with the use of oral CS and discover views on a short course tapering regime to manage mild to moderate flares of UC.

Method Patient views were assessed quantitatively and qualitatively via a Crohn's and Colitis UK led 'Patient involvement in research' event. The UC Steroid Questionnaire (UCSQ) poll 1 and 2 was developed based on patient and clinical input. Participants completed both UCSQ's online. Poll 1 was by the option of multiple choice and poll 2 indicating the degree of agreement with individual statements on a 10 point Likert scale. Further debriefing interviews with the same group of patients were conducted online evaluating the content, clarity, and relevance of the items.

Results Forty patients responded to UCSQ 1 and 49 to UCSQ 2. Approximately two-thirds (68\%) of patients had experienced significant side effects from oral CS use. Sleep disruption, mood disturbance and bone thinning were of highest concern (60\% vs $55 \%$ vs $55 \%)$. Overall only $53 \%$ were satisfied with the use of oral CS in managing a UC flare. Ninetyfive percent of patients said they would consider participating in a trial comparing a 1 month short course to a longer 2-3 months course of oral CS to manage a mild to moderate flare, whilst 5\% mentioned that they would not. The risk of suboptimal response and disease flare were amongst the main concerns elicited amongst those who were uncertain or said no.

Conclusion Significant side effects experienced by the majority of patients following oral CS use impacted on the opinions of many towards considering a shorter CS tapering regime to manage a mild to moderate flare of UC. With better understanding of patient experiences to steroid use, we highlight the need in defining the optimal oral CS tapering regime to reduce the risk of potentially avoidable disease progression.

\section{REFERENCES}

1. Truelove SC, Jewell DP. Intensive intravenous regimen for severe attacks of ulcerative colitis. Lancet. 1974; 1:1067-1070. 